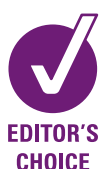

CHOICE

\title{
Endosaccular treatment of 113 cavernous carotid artery aneurysms
}

\author{
Armen Choulakian, Doniel Drazin, Michael J Alexander
}

Department of Neurosurgery, Cedars-Sinai Medical Center, Los Angeles, California, USA

\section{Correspondence to} Dr M J Alexander, Department of Neurosurgery, Cedars-Sinai Medical Center, 8631 W Third St, Suite 800E, Los Angeles, CA 90048, USA:

michael.alexander@cshs.org

Received 26 May 2010 Revised 10 August 2010 Accepted 11 August 2010

\begin{abstract}
Objective Cavernous carotid aneurysms (CCAs) can present with visual symptoms or with subarachnoid hemorrhage (SAH). As surgical treatment of these aneurysms can be technically challenging, endovascular management has emerged as the preferred treatment modality.
\end{abstract}

Methods A retrospective review was conducted of 113 patients who underwent endosaccular treatment for CCAs. Presenting symptoms, aneurysm size, use of stent assistance, rate of thromboembolic complications, presence of SAH and angiographic follow-up were reviewed.

Results 29 patients (26\%) with CCAs presented with diplopia due to cranial nerve palsies. Mean aneurysm size in this group was $17 \mathrm{~mm}$. Three patients (2.6\%) presented with $\mathrm{SAH}$ with a mean aneurysm size of $15.3 \mathrm{~mm}$. Mean length of stay for ruptured versus nonruptured aneurysms was 11.7 and 1.7 days, respectively. Clinically significant thromboembolic complications occurred in four cases (3.5\%). Stent assistance was required in 53 cases (47\%). Of the 86 patients (76\%) returning for follow-up angiography (mean 6.2 months), $58(75 \%)$ had no residual aneurysm and $14(12 \%)$ showed regrowth. Thirteen patients $(11.5 \%)$ underwent repeat endovascular treatment.

Conclusions CCAs commonly produce diplopia and cranial nerve palsies when a critical size is reached (mean $17 \mathrm{~mm}$ in our series). Aneurysm obliteration with internal carotid artery preservation is the preferred treatment modality and can be accomplished with coil embolization with or without stent assistance. Although recurrence and retreatment can occur, the thromboembolic risk of endovascular treatment is low. Consideration should be given to treatment of asymptomatic CCAs $15 \mathrm{~mm}$ or larger due to potential risks of cranial neuropathy and $\mathrm{SAH}$.

\section{INTRODUCTION}

Cavernous carotid aneurysms (CCAs) account for $2-9 \%$ of all intracranial aneurysms. ${ }^{1}$ The majority of these aneurysms are discovered incidentally with non-invasive imaging during work-up for unrelated intracranial disorders. Occasionally CCAs can become symptomatic either from a mass effect on adjacent structures in the cavernous sinus or from rupture resulting in subarachnoid hemorrhage (SAH) or carotid-cavernous fistula (CCF) formation. Treatment is necessary for symptomatic lesions to prevent potentially permanent cranial neuropathies or re-rupture. Most asymptomatic lesions do not require treatment as they pose a very small risk of severe complications. Larger CCAs, those which demonstrate growth on serial imaging studies, or those which have extended into the maxillary sinus or subarachnoid space, may be considered for treatment before they become symptomatic. ${ }^{2}$ The minimum size of asymptomatic CCAs for which treatment is recommended has not yet been established. Due to the potential morbidity and technical challenges from surgical approaches to the cavernous sinus, endovascular treatment has become the primary treatment modality for CCAs. Aneurysm obliteration with internal carotid artery (ICA) preservation has emerged as the preferred strategy with the advent of detachable coils and intracranial stents.

\section{METHODS AND MATERIALS}

A retrospective review was conducted of 113 patients who underwent endosaccular treatment for CCAs from 2001 to 2010. Patients with deliberate parent artery occlusion were excluded from the analysis. Presenting symptoms, aneurysm size, use of stent assistance, rate of thromboembolic complications, presence of SAH and angiographic follow-up were reviewed.

\section{RESULTS}

Twenty-nine patients (26\%) with CCAs presented with diplopia due to cranial nerve palsies. Mean aneurysm size in this group was $17 \mathrm{~mm}$. Three patients $(2.6 \%)$ presented with SAH with a mean aneurysm size of $15.3 \mathrm{~mm}$. Mean length of stay for ruptured versus non-ruptured aneurysms was 11.7 and 1.7 days, respectively. Clinically significant thromboembolic complications occurred in four cases (3.5\%). Stent assistance was required in 53 cases $(47 \%)$. Of the 86 patients $(76 \%)$ returning for follow-up angiography (mean 6.2 months), 58 (75\%) had no residual aneurysm and 14 (12\%) showed regrowth. Thirteen patients $(11.5 \%)$ underwent repeat endovascular treatment for either significant residual aneurysm or aneurysm regrowth. Representative cases are shown in figures $1-3$.

\section{DISCUSSION}

CCAs can be found incidentally on neuroimaging or can become symptomatic in a variety of ways, including rupture. Although rare, CCAs can present with rupture into either the cavernous sinus or subarachnoid space. Direct CCFs can result if a CCA ruptures into the cavernous sinus. In one study, the incidence of CCF by a ruptured CCA was $1.5 \%{ }^{3}$ If a CCA erodes through the sphenoid bone into the maxillary sinus, rupture may result in catastrophic epistaxis. Extension of a CCA into the subarachnoid space, occasionally seen as 'dural waisting' on cerebral angiography, can predispose to $\mathrm{SAH}^{4}$ The risk of SAH from CCAs is relative to 
Figure 1 Patient No 1 presented with headaches. (A) Pre-procedure right internal carotid artery (ICA) angiogram demonstrated a $14 \mathrm{~mm}$ cavernous carotid artery aneurysm. The patient underwent coil embolization without stent assistance. (B) Post-procedure right ICA angiogram demonstrated complete occlusion of the aneurysm.
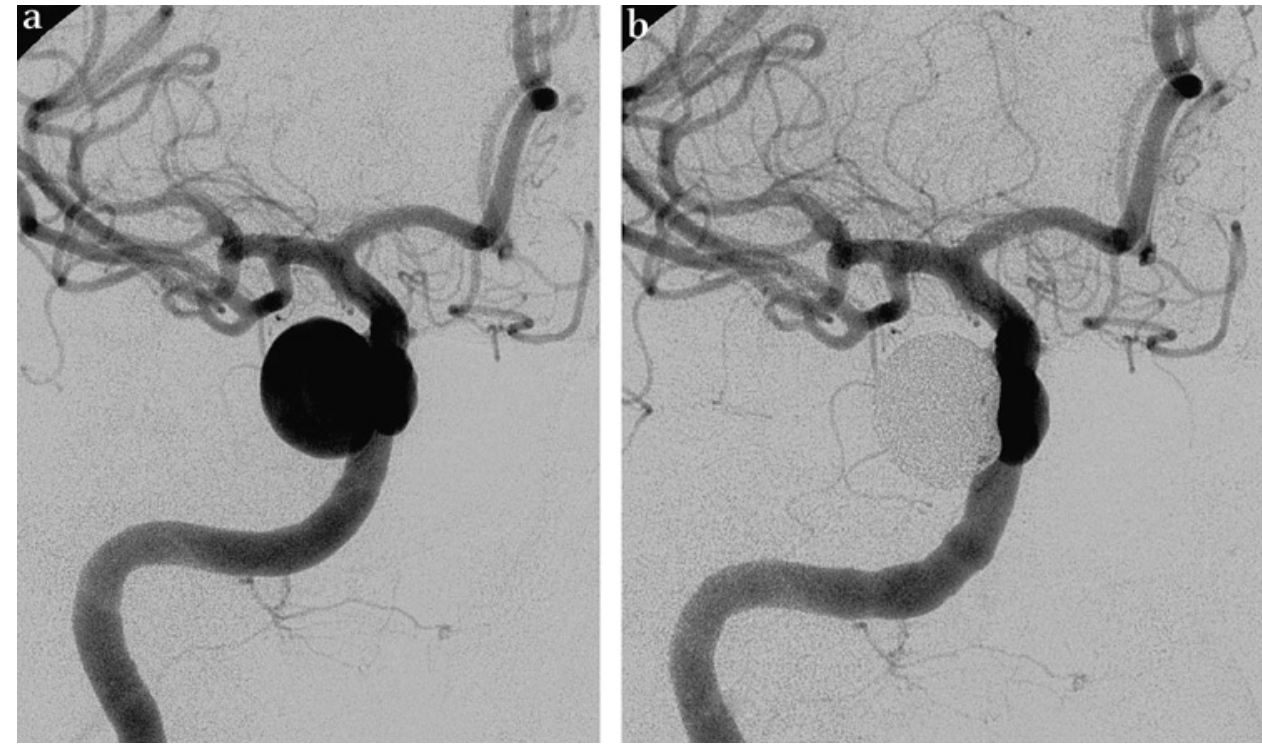

size, with aneurysms $<12 \mathrm{~mm}$ having a $0 \%$ rupture risk over 5 years while those $>25 \mathrm{~mm}$ have a $6.4 \%$ rupture risk over the same time period. ${ }^{5}$ CCAs $13-24 \mathrm{~mm}$ in size have a 5 year rupture risk of $3.0 \% .^{5}$ In our series, three patients with CCAs presented with $\mathrm{SAH}$ and had no other aneurysms identified on digital subtraction angiography. In all three cases the aneurysm neck began in the cavernous carotid artery but the aneurysm was transitional into the subarachnoid space.

More commonly, CCAs present with either pain or visual symptoms, especially when they reach a giant size. Pain is usually facial or retro-orbital in location but generalized headache can also be reported. Diplopia is the most common visual symptom and results from paresis of the third, fourth or sixth cranial nerves. Involvement of any of the divisions of the fifth cranial nerve can result in analgesia or hypalgesia in that territory. Any combination of cranial nerves in the cavernous sinus can become affected and the degree of symptoms is highly variable. $^{6}$

Symptomatic CCAs, specifically those which have ruptured or are causing cranial neuropathies, have traditionally undergone treatment to prevent rebleeding or permanent oculoparesis and vision loss. ${ }^{2}$ Multiple studies have found better outcomes in improvement or stabilization of pain symptoms and neuroophthalmologic deficits in patients with CCAs who receive treatment versus those who do not. ${ }^{7} 8$ One study however found that treatment had a significant affect only on patients presenting with pain but not on patients presenting with diplopia.? Follow-up visual analysis was not included in our study as only 86 patients $(76 \%)$ had descriptions of their visual symptoms after treatment at angiographic follow-up. Inclusion of data from this select group of patients would not be an accurate reflection of the 113 aneurysms treated in this series, as it is perceived that patients with recurrent or residual symptoms are more likely to follow-up, presenting a selection bias.

Traditionally, asymptomatic lesions, especially when small, have not been treated as they are of low risk to the patient. ${ }^{10} 11$ However, consideration can be given to treatment of large asymptomatic CCAs in the hope of preventing future symptoms, because as these aneurysms enlarge they can produce a mass effect on structures of the cavernous sinus and
Figure 2 Patient No 2 presented with a 2 month history of right third and sixth nerve palsies. (A) Pre-procedure right internal carotid artery (ICA) angiogram demonstrated a $20 \mathrm{~mm}$ cavernous carotid artery aneurysm. The patient underwent coil embolization with Neuroform (Boston Scientific, Natick, Massachusetts, USA) stent assistance. (B) Post-procedure right ICA angiogram demonstrated a good result.
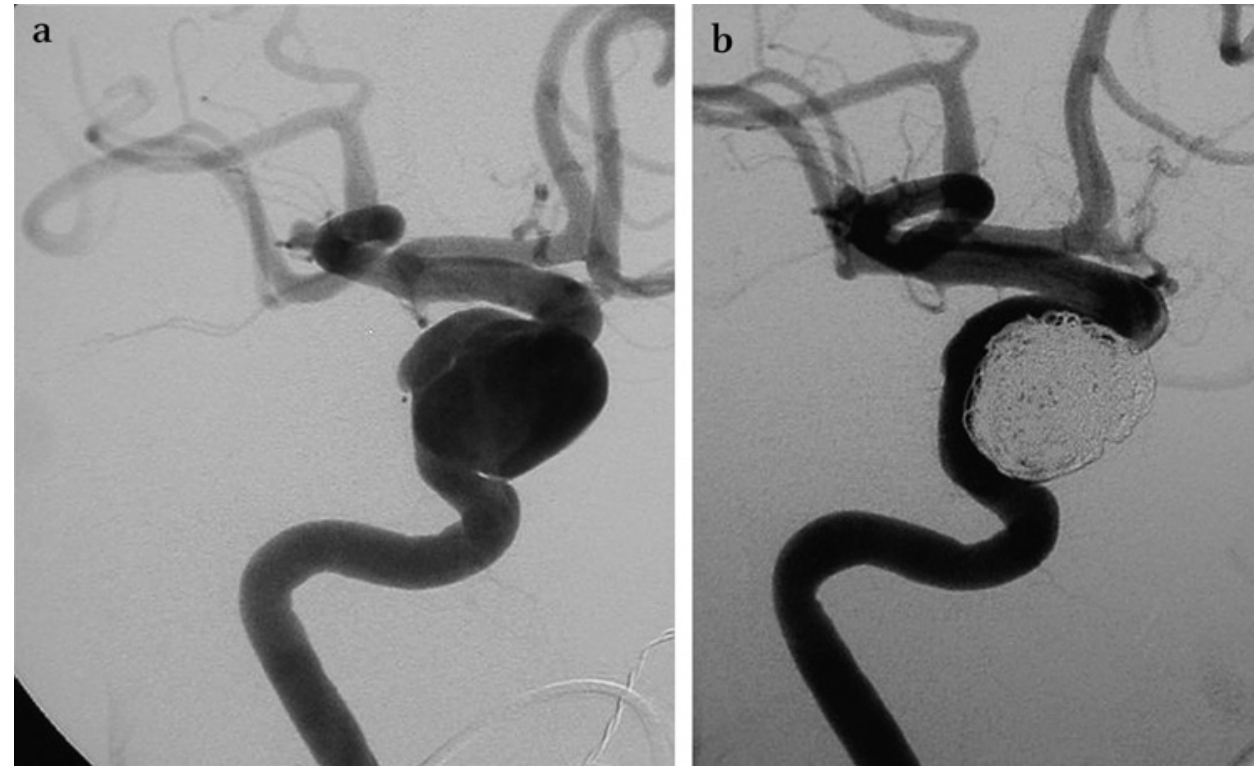

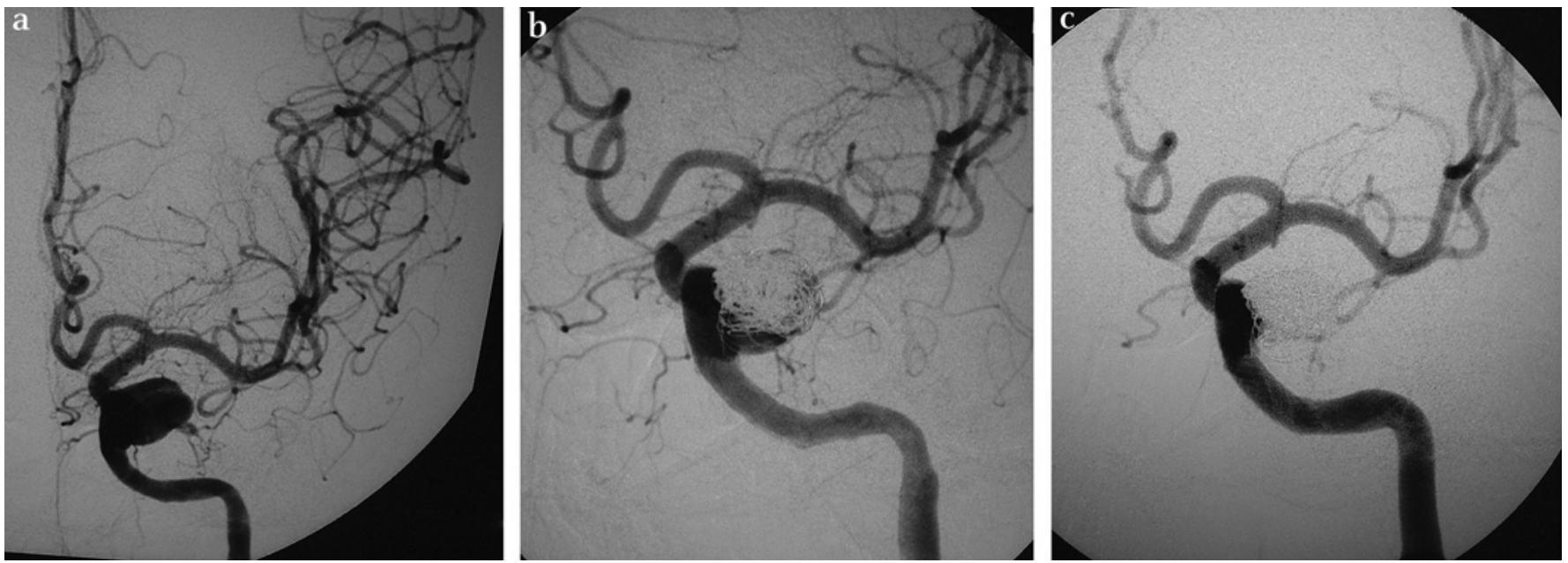

Figure 3 Patient No 3 presented with aphasic transient ischemic attacks and a history of a motor vehicle collision 20 years earlier. (A) Pre-procedure left internal carotid artery (ICA) angiogram demonstrated a $20 \mathrm{~mm}$ dissecting cavernous carotid artery aneurysm. The patient underwent coil embolization with Magic Wallstent (Boston Scientific, Natick, Massachusetts, USA) assistance. (B) Immediate post-procedure left ICA angiogram demonstrated residual aneurysm filing. (C) Six month follow-up left ICA angiogram demonstrated progressive occlusion of the residual aneurysm.

potentially lead to cranial neuropathies. In addition, large CCAs can remodel the boney confines of the cavernous sinus and grow into the subarachnoid space or maxillary sinus leading to potential SAH or severe epistaxis if they rupture. In our series, the mean size of CCAs presenting with cranial nerve palsies was $17 \mathrm{~mm}$ and CCAs presenting with SAH was $15.3 \mathrm{~mm}$.

Surgical approaches for direct repair of CCAs are technically challenging, especially for larger aneurysms, and carry with them a high risk of cranial nerve morbidity. For these reasons endovascular approaches have largely supplanted surgical management as the primary treatment modality for CCAs. Endovascular sacrifice of the ICA, initially with detachable balloons and more recently with electrolytically detachable coils, has been shown to be an effective method of excluding CCAs from the cerebral circulation. ${ }^{12-15}$ There is however up to a $25 \%$ risk of developing cerebral infarctions if the ICA is sacrificed without determining whether adequate collateral cerebral blood flow is present. ${ }^{16}$ Balloon test occlusion of the ICA followed by either single photon emission CT or other imaging can help identify which patients are at high risk for developing cerebral infarctions and allow for an extracranial to intracranial bypass to be performed prior to ICA sacrifice. ${ }^{17} 18$ Alternatively, balloon test occlusion with hypotensive challenge and clinical examination has also been shown to be an acceptable means of accessing the collateral circulation without the use of quantitative imaging. ${ }^{19}$

Although ICA sacrifice is at times necessary, aneurysm obliteration with ICA preservation is the most desirable method of treatment. This can be accomplished in smaller CCAs with narrow necks using detachable coils alone. ${ }^{20}$ Unfortunately, many CCAs that require treatment are large and can have extremely wide necks, sometimes even encompassing the entire ICA. Balloon remodeling of the aneurysm neck can be used in the treatment of some wide necked CCAs but is of limited use when the aneurysm is fusiform or has no discernable neck. With the addition of neurovascular stents to the neurointerventional armamentarium, even these extremely challenging lesions can be treated with ICA preservation. Stent assisted coiling, either with the open cell Neuroform (Boston Scientific, Natick, Massachusetts, USA) or closed cell Enterprise (Codman Neurovascular, Raynham, Massachusetts, USA) stents, allows reconstruction of the ICA wall followed by embolization of the aneurysm. This is currently our preferred method of treatment for large wide necked CCAs with nearly half of the cases in our series requiring stent assistance (47\%). Emerging endovascular treatment options for these lesions include easily navigateable covered stent grafts, stand alone flow diverters like the Pipeline Embolization Device (ev3, Irvine, California, USA), and liquid embolic agents such as Onyx (ev3).

The rates of incomplete treatment, recurrence and retreatment are high when treating large CCAs via the endovascular route with ICA preservation. Specifically, we had a $25 \%$ rate of residual aneurysm on follow-up imaging with $12 \%$ of patients showing regrowth and $11.5 \%$ being significant enough to require retreatment. However, the risk of endovascular treatment of CCAs is low, with only $3.5 \%$ clinically significant thromboembolic events occurring in our series.

\section{CONCLUSION}

CCAs commonly produce diplopia and cranial nerve palsies when a critical size is reached (mean $17 \mathrm{~mm}$ in our series). Aneurysm obliteration with ICA preservation is the preferred treatment modality and can be accomplished with coil

\section{Key messages}

- Symptomatic cavernous carotid aneurysms (CCAs) can present with pain, visual symptoms or rupture.

- Endovascular therapy is the primary treatment modality for symptomatic CCAs due to the technical difficulties and morbidity associated with surgical approaches to the cavernous sinus.

- Aneurysm embolization and internal carotid artery preservation, with or without stent assistance, can be accomplished with low morbidity and with acceptable recurrence and retreatment rates.

- Consideration should be given to treatment of asymptomatic CCAs $15 \mathrm{~mm}$ or larger due to potential risks of cranial neuropathy and subarachnoid hemorrhage. 
embolization with or without stent assistance. Although recurrence and retreatment can occur, the thromboembolic risk of endovascular treatment is low. Consideration should be given to treatment of asymptomatic CCAs $15 \mathrm{~mm}$ or larger, or those that demonstrate progressive rapid growth due to potential risks of cranial neuropathy and SAH.

Competing interests MJA is a consultant for Boston Scientific (Natick, Massachusetts, USA) and Codman Neurovascular (Raynham, Massachusetts, USA) $\mathrm{AC}$ is the recipient of Boston Scientific and Cordis Endovascular (Bridgewater, New Jersey, USA) fellowship training grants.

Patient consent Detail has been removed from this case description to ensure anonymity. The editors and reviewers have seen the detailed information available and are satisfied that the information backs up the case the authors are making.

Provenance and peer review Not commissioned; externally peer reviewed.

\section{REFERENCES}

1. Eddleman CS, Hurley MC, Bendok BR, et al. Cavernous carotid aneurysms: to treat or not to treat? Neurosurg Focus 2009;26:E4

2. Linskey ME, Sekhar LN, Hirsch WL, et al. Aneurysms of the intracavernous carotid artery: natural history and indications for treatment. Neurosurgery 1990;26:933-8.

3. van Rooij WJ, Sluzewski M, Beute GN. Ruptured cavernous sinus aneurysms causing carotid cavernous fistula: incidence, clinical presentation, and outcome. AJNR Am J Neuroradiol 2006;27:185-9

4. White JA, Horowitz MB, Samson D, et al. Dural waisting as a sign of subarachnoid extension of cavernous carotid aneurysms: a follow-up case report. Surg Neurol 1999:52:607-10.

5. Wiebers D0, Whisnant JP, Huston J III, et al. Unruptured intracranial aneurysms: natural history, clinical outcome, and risks of surgical and endovascular treatment. Lancet 2003;362:103-10.

6. Hahn CD, Nicolle DA, Lownie SP, et al. Giant cavernous carotid aneurysms: clinical presentatio in fifty-seven cases. J Neuroophthalmol 2000;20:253-8.
7. de Vasconcellos LP. Flores JA, Veiga JC et al. Presentation and treatment of carotid cavernous aneurysms. Arq Neuropsiquiatr 2008;66:189-93.

8. Goldenberg-Cohen N, Curry C, Miller NR, et al. Long term visual and neurological prognosis in patients with treated and untreated cavernous sinus aneurysms. J Neurol Neurosurg Psychiatry 2004;75:863-7.

9. Stiebel-Kalish $\mathbf{H}$, Kalish $\mathrm{Y}$, Bar-On RH, et al. Presentation, natural history, and management of carotid cavernous aneurysms. Neurosurgery 2005;57:850-7.

10. Inagawa T. Follow-up study of unruptured aneurysms arising from the C3 and C4 segments of the internal carotid artery. Surg Neurol 1991:36:99-105.

11. Kupersmith MJ, Hurst R, Berenstein A, et al. The benign course of cavernous carotid artery aneurysms. J Neurosurg 1992;77:690-3.

12. Kupersmith MJ, Berenstein A, Choi IS, et al. Percutaneous transvascula treatment of giant carotid aneurysms: neuro-ophthalmologic findings. Neurology 1984;34:328-35.

13. Higashida RT, Halbach VV, Dowd C, et al. Endovascular detachable balloon embolization therapy of cavernous carotid artery aneurysms: results in 87 cases. J Neurosurg 1990;72:857-63.

14. Bavinzski G, Killer M, Ferraz-Leite $\mathrm{H}$, et al. Endovascular therapy of idiopathic cavenrous aneurysms over 11 years. AJNR Am J Neuroradiol 1998;19:559-65.

15. van der Schaaf IC, Brilstra EH, Buskens E, et al. Endovascular treatment of aneurysms in the cavenrous sinus: a systematic review on balloon occlusion of the parent vessel and embolization with coils. Stroke 2002;33:313-18.

16. Linskey ME, Jungreis CA, Yonas $\mathrm{H}$, et al. Stroke risk after abrupt internal carotid artery sacrifice: accuracy of preoperative assessment with balloon test occlusion and stable xenon-enhanced CT. AJNR Am J Neuroradiol 1994;15:829-43.

17. Field $\mathbf{M}$, Jungreis CA, Chengelis $\mathbf{N}$, et al. Symptomatic cavenrous sinus aneurysms: management and outcome after carotid occlsuion and selective cerebral revascularization. AJNR Am J Neuroradiol 2003;24:1200-7.

18. Kai Y, Hamada J, Morioka M, et al. Treatment strategy for giant aneurysms in the cavernous portion of the internal carotid artery. Surg Neurol 2007;67:148-55

19. Standard S, Ahuja A, Guterman LR, et al. Balloon test occlusion of the internal carotid artery with hypotensive challenge. AJNR Am J Neuroradiol 1995; 16:1453-8.

20. Halbach VV, Higashida RT, Dowd CF, et al. Cavernous internal carotid artery aneurysms treated with electrolytically detachable coils. J Neuroophthalmol 1997:17:231-9. 